\title{
Neural activation associated with corrective saccades during tasks with fixation, pursuit and saccades
}

\author{
Sven Haller $\cdot$ David Fasler $\cdot$ Sabine Ohlendorf $\cdot$ \\ Ernst W. Radue $\cdot$ Mark W. Greenlee
}

Received: 29 May 2006 / Accepted: 21 July 2007 / Published online: 24 August 2007

(C) Springer-Verlag 2007

\begin{abstract}
Corrective saccades are small eye movements that redirect gaze whenever the actual eye position differs from the desired eye position. In contrast to various forms of saccades including pro-saccades, recentering-saccades or memory guided saccades, corrective saccades have been widely neglected so far. The fMRI correlates of corrective saccades were studied that spontaneously occurred during fixation, pursuit or saccadic tasks. Eyetracking was performed during the fMRI data acquisition with a fiber-optic device. Using a combined block and event-related design, we isolated the cortical activations associated with visually guided fixation, pursuit or saccadic tasks and compared these to the activation associated with the occurrence of corrective saccades. Neuronal activations in anterior inferior cingulate, bilateral middle and inferior frontal gyri, bilateral insula and cerebellum are most likely specifically associated with corrective saccades. Additionally, overlapping activations with the established pro-saccade and, to a
\end{abstract}

S. Haller $(\varangle) \cdot$ D. Fasler $\cdot$ E. W. Radue

Institute of Radiology, Department of Neuroradiology,

University Hospital Basel, CH 4031 Basel, Switzerland

e-mail: shaller@uhbs.ch

S. Haller

Institute of Radiology, Department of Diagnostic Radiology,

University Hospital Basel, CH 4031 Basel, Switzerland

S. Ohlendorf

Department of Neurology,

University Hospital Freiburg, 79106 Freiburg, Germany

M. W. Greenlee

Institute for Experimental Psychology,

University of Regensburg, 93053 Regensburg, Germany lesser extent, pursuit network were present. The presented results imply that corrective saccades represent a potential systematic confound in eye-movement studies, in particular because the frequency of spontaneously occurring corrective saccades significantly differed between fixation, pursuit and pro-saccades.

Keywords Pro-saccades - Corrective saccades . Pursuit eye movement $\cdot$ fMRI $\cdot$ BOLD
Abbreviations
ACG Anterior cingulate cortex
BOLD Blood oxygenation level dependent
CEF Cingulate eye field
DLPFC Dorsolateral prefrontal cortex
FEF Frontal eye fields
fMRI Functional magnetic resonance imaging
PEF Parietal eye fields
PCG Posterior cingulate cortex
SEF Supplementary eye field
$\mathrm{S} \quad$ Experimental condition pro-saccades
$\mathrm{P} \quad$ Experimental condition pursuit eye movement
CS Corrective saccades during pro-saccades
$\mathrm{CP} \quad$ Corrective saccades during pursuit eye movement
$\mathrm{CF}$ Corrective saccades during fixation

\section{Introduction}

The human and non-human primate visual system is characterized by the fovea, which contains a tightly packed mosaic of cone photoreceptors. The fovea being the center of highest visual acuity, is directed by conjugate eye movements to targets of interest, thereby achieving the best possible visual performance (Dodge 1903). There are two 
fundamental modes of conjugate eye movement. Saccades rapidly direct eye position from one target to the next. Saccades occur involuntarily, e.g., not only during reading (Huey 1900) or while scrutinizing a human face (Yarbus 1967) but also may be evoked voluntarily, when attention is directed to an object in a cluttered scene. Pursuit eye movement allows for the continuous tracking of a steadily moving target, and can only occur in the presence of a moving visual target (Pola and Wyatt 1991; Bennett and Barnes 2006). Fixation refers to a constant eye position with respect to a given target and may be considered as the baseline condition for the study of the oculomotor system. Neuronal activations associated with these fundamental types of eye movement differ significantly (Pierrot-Deseilligny et al. 2004).

Corrective saccades are small eye movements that may occur during saccades, pursuit and fixation. Corrective saccades are necessary whenever the actual eye position differs from the desired eye position. In order to perform corrective saccades, a neuronal circuitry first needs to detect the difference between actual and desired eye position and then compute an appropriate corrective eye movement. This process presumably differs depending on the on-going eye movement mode executed by the observer. During fixation, the visual target is stationary and it is sufficient to compute the difference between actual and desired eye position. During pro-saccades, the visual target is also stationary but the mismatch between actual and desired eye position must be taken into account when computing the next pro-saccade. During pursuit, the motion of the target must be anticipated and the corrective saccades, often referred to as catch-up saccades during pursuit, must be adapted to the speed and direction of the moving target accordingly.

Previous investigations assessed various forms of saccades including intentional saccades (internally triggered saccades towards a target already present), predictive saccades (target not yet present), memory guided saccades (target no longer visible), anti-saccades (saccade in the opposite direction of a visual target) or reflexive (visually guided) saccades often called 'pro-saccades' (for a review, Pierrot-Deseilligny et al. 2004). Corrective saccades, in contrast, have been widely neglected so far.

In the present investigation, we simultaneously acquired fMRI data (Ogawa et al. 1990; Belliveau et al. 1992; Friston et al. 1995) to assess neuronal activation, and used an MRI compatible eye tracker to assess eye movements (Kimmig et al. 1999). We tested the hypothesis that a specific neuronal circuitry mediates the control of corrective saccades. We expect that the pattern of activation evoked by corrective saccades will partially overlap yet will differ from the networks subserving pro-saccades, pursuit and fixation.

\section{Materials and methods}

Subjects

The study was approved by the local ethics committee. Fourteen subjects ( 7 females, mean age 28.9 years, \pm 11.8 years standard deviation) gave their written informed consent prior to inclusion in the study and were non-smokers, had normal vision and no history of medical, neurological or psychiatric disorders.

Stimuli and task

A block-design fMRI paradigm was implemented with conditions fixation (F), saccades (S) and pursuit (P). Each condition lasted $20 \mathrm{~s}$. One cycle consisted of a trial sequence: fixation, saccades, fixation, pursuit (FSFP). Each run consisted of five cycles and each subject performed two runs. The stimuli were back-projected onto a translucent screen mounted to the table of the MR imager and mirrors were attached to the head coil. The video projector (PLUS Vision, Tokyo, Japan) with a resolution of $1,024 \times 768$ pixels was placed in the control room. The horizontal angle subtended by the entire screen was approximately $23^{\circ}$. The imaging room was darkened during the experiment. The subject's head was immobilized, and noise protection was provided.

In the fixation condition $(\mathrm{F})$, a stationary dot of $0.5^{\circ}$ diameter was presented in the center of the visual field. In the saccade condition $(\mathrm{S})$, saccades were performed on average every $500 \mathrm{~ms}$ jittered $\pm 100 \mathrm{~ms}$. Saccades were randomly performed to targets presented to the far right (equivalent to $11.5^{\circ}$ ), to the intermediate right $\left(5.75^{\circ}\right)$, to the intermediate left or to the far left. In the pursuit condition (P), the dot performed a smooth, sinusoidal movement to the far left to the far right, starting at the center with a frequency of $0.3 \mathrm{~Hz}$. The speed of the pursuit target was maximal at the central location with a peak velocity of 21.7 per second.

Data acquisition

MR imaging was performed with a 1.5-Tesla scanner (Sonata; Siemens Medical Systems, Erlangen, Germany), and transverse functional T2*-weighted MR images were obtained with an echo-planar single-shot pulse sequence. The matrix size was $64 \times 64$ (field of view, $192 \times 192 \mathrm{~mm}$ ). Twenty-five sections were acquired (4.0$\mathrm{mm}$ section thickness, 1-mm gap), which covered the whole brain. The resulting resolution was $3 \times 3 \times 5$-mm voxels. Repetition time was $2.5 \mathrm{~s}$, flip angle was $90^{\circ}$, and echo time was $50 \mathrm{~ms}$. After functional MR imaging, highspatial-resolution data were acquired (1-mm iso-voxel T1weighted magnetization-prepared rapid gradient echo; 
matrix, $256 \times 256$; 176 sections; 1,900 ms repetition time and $3.68 \mathrm{~ms}$ echo time) for cortex normalization and cortex surface reconstruction. The head coil used was a standard SIEMENS single-channel birdcage, because this head coil had sufficient space to mount the eye tracker.

Horizontal eye position was recorded using a fiber-optic, MR compatible, IRlight limbus reflection eye tracker (Cambridge Research Systems, http://www.crsltd.com) at a sample rate of $500 \mathrm{~Hz}$. We could resolve saccades that had an amplitude of $>0.8^{\circ}$ in all subjects.

\section{Data analysis}

Anatomical and functional images were analyzed using BrainVoyager QX (Brain Innovation, http://www.brainvoyager.com). Anatomical scans were segmented for identification of the white-gray matter boundary used for cortical surface reconstruction and cortex-based statistics. Concerning the functional time series, the first three of 160 volumes were discarded from further analysis to avoid non-steady state saturation effects. Pre-processing consisted of three-dimensional motion correction, slice scan time correction, Gaussian spatial filtering (full width half maximum $4 \mathrm{~mm}$ ), high-pass temporal filtering (three cycles per time course) and transformation into standard space (Talairach and Tournoux 1988). Because we assume that corrective saccades depend on the on-going eye movement mode, as discussed in the "Introduction", we did not generate a single corrective saccades predictor/regressor. Instead, we consider it more appropriate to separate corrective saccades occurring during fixation, pro-saccades and pursuit into $\mathrm{CF}, \mathrm{CS}$ and $\mathrm{CP}$, respectively. Consequently, we must take into account smaller expected individual effects for $\mathrm{CF}, \mathrm{CS}$ and $\mathrm{CP}$ compared to a general linear model with a single corrective saccades predictor/regressor. Therefore, we performed fixed-effects GLM analyses. The presented results thus refer to the investigated sample. This GLM included five predictors/regressors. Three event-related predictors $\mathrm{CF}, \mathrm{CS}$ and $\mathrm{CP}$ were defined for each run of each subject by the individual onset of corrective saccades during fixation, pro-saccades and pursuit, respectively, analyzed from the eye-tracker recordings in MATLAB (The MathWorks, http://www.mathworks.com). Additionally, we defined block-design predictors that represent the eyemovement tasks fixation (F), pro-saccades (S) and pursuit (P). In order not to over-determine the GLM, we defined only two block-design predictors $\mathrm{P}$ and $\mathrm{S}$ based on the known sequence of experimental conditions. Condition $\mathrm{F}$ was not modeled separately because there are only three conditions F, S and P. Consequently, condition F is immanent in the model as non-S and non-P. F might be regarded as baseline condition. All predictors/regressors were convolved with a hemodynamic reference function consisting of two superimposed gamma functions (Boynton et al. 1996). Statistical thresholding was corrected for multiple comparisons based on the false discovery rate (FDR) (Genovese et al. 2002) at a false-positive probability of $\mathrm{q}(\mathrm{FDR})<0.05$. This corresponded to $t>2.27$ for pro-saccades, $t>2.63$ for pursuit, $t>2.75$ for pro-saccades versus pursuit, $t>2.71$ for CS and $t>3.11$ for CF. The spatial extent threshold was $200 \mathrm{~mm}^{3}$.

Statistical analysis of the eye traces was performed using PRISM (GraphPad, http://www.graphpad.com). Individual frequencies of corrective saccades were determined. Because the frequency of CF, CS and CP of the 14 subjects exhibited a non-normal distribution according to the Kolmogorov-Smirnov Test $(P<0.01)$, we used non-parametric, repeated-measures Friedman test to test for differences between conditions with respect to saccade frequencies. Post-hoc pair-wise comparison was performed using Dunn's Multiple Comparison Test.

Blinks, drifts and other artifacts were detected and eliminated from the oculomotor data. We only classified saccades as corrective saccades if their amplitude fell within $0.8^{\circ}$ and $8^{\circ}$. Usually these occurred as single events and led to central fixation of the target stimulus.

To validate the presented analysis and to control for the possible confounding effects of the activation evoked by the primary (pro-saccades, pursuit) task, we reanalyzed the data but varied the onset of corrective saccades thereby creating new event-related predictors pseudo-CF, pseudo-CS and pseudo-CP. This was achieved by randomly assigning individual event files to 'wrong' subjects, for example, events (corrective saccades) of subject 1 were assigned to subject 2 , etc. This very simple approach has the advantage that when considering the GLM group analysis, the eventrelated properties such as frequency, temporal distribution, between-subject variation of events, etc. are identical to the 'correct' analysis. The block-design regressors $\mathrm{S}$ and $\mathrm{P}$ were unchanged. We analyzed four randomly chosen variants of the numerous possibilities.

\section{Results}

\section{Behavioral data}

The frequency of corrective saccades during fixation $(\mathrm{CF})$ was $0.152 \pm 0.155 / \mathrm{s}$ (mean of individual frequency \pm standard deviation), $0.552 \pm 0.337$ during pro-saccades (CS) and $1.416 \pm 0.309$ during pursuit $(\mathrm{CP})$. These differences are significant $(P<0.0001)$. Post-hoc comparison revealed significant differences between all pairs: $C F$ versus CS $P<0.05$, CF versus CP $P<0.001$ and $\mathrm{CS}$ versus $\mathrm{CP}$ $P<0.05$. The majority of corrective saccades during pursuit were catch-up saccades. During fixation, the majority 
of corrective saccades were a sequence of corrective saccade away from the center of gaze, followed by a re-centering saccade that is also known as square-wave jerk. Figure 1 illustrates an example of eye-tracker recording and corrective saccades during fixation (A), pro-saccades (B) and pursuit (C).

Pro-saccades and pursuit

Activations associated with pro-saccades were present in a distributed network including bilateral frontal eye fields
(FEF), supplementary eye fields (SEF), parietal eye fields (PEF) and primary visual area (Fig. 2a, Table 1). Pursuit eye movement was associated with a distributed network, which also included FEF, SEF and PEF and the cerebellum (Fig. 2a, Table 1).

The direct comparison of pro-saccades and pursuit (Fig. 2b, Table 2) revealed stronger activations during prosaccades compared to pursuit within the known pro-saccade-associated network including bilateral FEF, SEF and PEF. Stronger activations for pursuit compared to pro-saccades were present in the known pursuit-associated net-

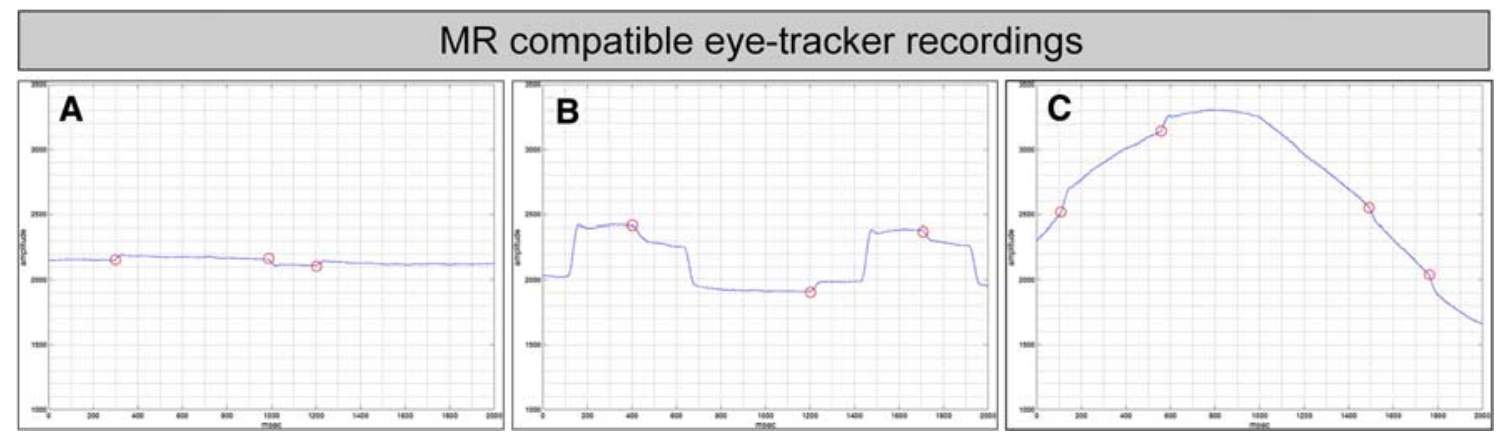

Fig. 1 Depiction of an example of eye-tracker recordings of one volunteer during fixation (a), pro-saccades (b) and pursuit (c). Corrective saccades occur during all conditions and are marked with red circles. Note that pro-saccade targets were randomly presented to left or right, half or full amplitude. The example b displays two consecutive prosaccades in the same direction with half amplitude. $\mathrm{X}$-axis indicates time (in $\mathrm{ms}$ ), Y-axis indicates raw eye position (arbitrary units)

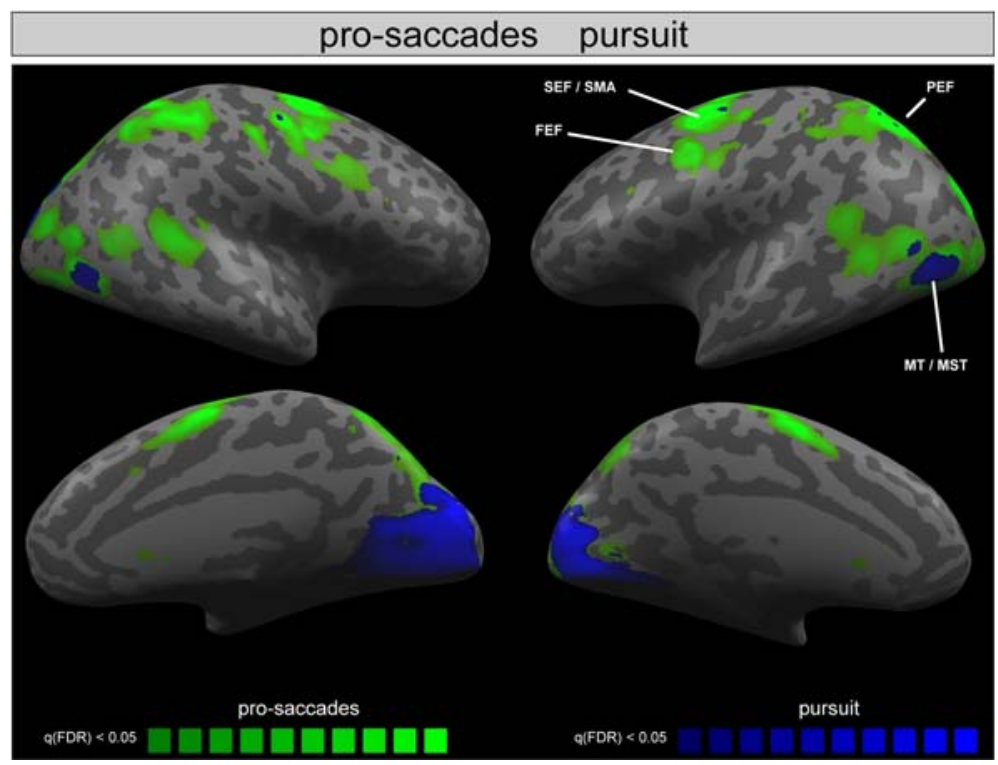

Fig. 2 Pro-saccades (S, green) and pursuit eye movement (P, blue) evoke activations in a distributed and largely overlapping network including FEF, SEF, PEF and the human homologue of $M T / M S T$, which is in general more prominently activated for S except MT/MST. The direct statistical comparison (Table 1) confirms this observation and reveals additional activations for $\mathrm{S}$ compared to $\mathrm{P}$ in bilateral middle frontal gyri, left inferior frontal gyrus and left anterior insula.
Conversely, $\mathrm{P}$ compared to $\mathrm{S}$ (blue) evoked stronger activations in bilateral cerebellum. Activations are superimposed on the inflated MNI reference brain in Talairach space; activations are based on a fixed-effects GLM corrected for multiple comparisons at $\mathrm{q}(\mathrm{FDR})<0.05$. Essential anatomical regions are labelled: $F E F$ frontal eye fields, $P E F$ posterior eye fields, $S E F$ supplementary eye fields, SMA supplementary motor area 
Table 1 Activation clusters for the analysis of S, P and the direct comparison of S versus $\mathrm{P}$ based on general linear models corrected for multiple comparisons at $\mathrm{q}(\mathrm{FDR})<0.05$

\begin{tabular}{|c|c|c|c|c|c|c|c|}
\hline \multicolumn{8}{|c|}{ A pro-saccades and pursuit } \\
\hline Size & $t \max$ & TAL X & TAL Y & TAL Z & Side & Anatomic region & $\mathrm{BA}$ \\
\hline \multicolumn{8}{|c|}{ Pro-saccades } \\
\hline \multirow[t]{19}{*}{188,899} & 25.3 & $0.89 \pm 26$ & $-64 \pm 14$ & $11 \pm 22$ & Left/right & Superior parietal lobule including PEF & 7 \\
\hline & & & & & & Precuneus & 7 \\
\hline & & & & & & Inferior parietal lobule & 40 \\
\hline & & & & & & Cuneus & 171819 \\
\hline & & & & & & Middle occipital gyrus & 1819 \\
\hline & & & & & & Inferior occipital gyrus & 18 \\
\hline & & & & & & Lingual gyrus & 171819 \\
\hline & & & & & & Superior temporal gyrus & 22 \\
\hline & & & & & & Middle temporal gyrus & 21 \\
\hline & & & & & & Inferior temporal gyrus & 37 \\
\hline & & & & & & Cerebellum & NA \\
\hline & 25.3 & -8 & -70 & 5 & Left & Lingual gyrus & 18 \\
\hline & 20.8 & 11 & -77 & 5 & Right & Lingual gyrus & 18 \\
\hline & 15.1 & -28 & -56 & 48 & Left & Superior parietal lobule & 7 \\
\hline & 11.8 & -42 & -67 & 2 & Left & Middle temporal gyrus & 37 \\
\hline & 11.7 & 21 & -58 & 51 & Right & Superior parietal lobule & 7 \\
\hline & 11.5 & 42 & -61 & 3 & Right & Middle temporal gyrus & 37 \\
\hline & 11.5 & -26 & -75 & 21 & Left & Cuneus & 18 \\
\hline & 10.3 & 23 & -79 & 23 & Right & Cuneus & 18 \\
\hline \multirow[t]{7}{*}{38,716} & 12.9 & $-0.41 \pm 30$ & $-6.7 \pm 6.6$ & $47 \pm 9.6$ & Left/right & Superior, middle frontal gyrus including FEF & 6946 \\
\hline & & & & & & Medial frontal gyrus including SMA, SEF & 6 \\
\hline & & & & & & Anterior cingulate & 2432 \\
\hline & & & & & & Inferior frontal gyrus & 44 \\
\hline & 12.9 & 26 & -10 & 52 & Right & Middle frontal gyrus & 6 \\
\hline & 11.8 & -28 & -9 & 47 & Left & Middle frontal gyrus & 6 \\
\hline & 10.0 & -2 & -4 & 54 & Left/right & Medial frontal gyrus & 6 \\
\hline 790 & 3.1 & $-4.2 \pm 5.8$ & $15 \pm 4.6$ & $3.1 \pm 3$ & Left/right & Caput of caudate nucleus & NA \\
\hline 273 & 3.2 & $-38 \pm 2.1$ & $9.9 \pm 1.7$ & $22 \pm 1.7$ & Left & Middle frontal gyrus & 946 \\
\hline \multicolumn{8}{|l|}{ Pursuit } \\
\hline \multirow[t]{7}{*}{73,364} & 17.5 & $2.1 \pm 13$ & $-69 \pm 12$ & $5.5 \pm 13$ & Left/right & Cuneus & 171819 \\
\hline & & & & & & Posterior cingulate gyrus & 23293031 \\
\hline & & & & & & Lingual gyrus & 171819 \\
\hline & & & & & & Parahippocampal gyrus & 2730 \\
\hline & & & & & & Cerebellum & NA \\
\hline & 17.5 & -9 & -71 & 6 & Left & Lingual gyrus & 18 \\
\hline & 17.2 & 10 & -68 & 8 & Right & Lingual gyrus & 18 \\
\hline \multirow[t]{2}{*}{2,500} & 6.8 & $-42 \pm 3.8$ & $-66 \pm 3.9$ & $3.5 \pm 4$ & Left & Middle temporal gyrus & 37 \\
\hline & & & & & & Middle occipital gyrus & 19 \\
\hline \multirow[t]{2}{*}{1,234} & 5.7 & $41 \pm 2.3$ & $-62 \pm 3.2$ & $3.5 \pm 4.1$ & Right & Middle temporal gyrus & 37 \\
\hline & & & & & & Middle occipital gyrus & 19 \\
\hline \multirow[t]{2}{*}{249} & 3.7 & $37 \pm 1.9$ & $-11 \pm 1.6$ & $49 \pm 2$ & Right & Precentral gyrus & 4 \\
\hline & & & & & & Middle frontal gyrus & 6 \\
\hline
\end{tabular}


Table 1 continued

\begin{tabular}{|c|c|c|c|c|c|c|c|}
\hline \multicolumn{8}{|c|}{ A pro-saccades and pursuit } \\
\hline Size & $t \max$ & TAL $\mathrm{X}$ & TAL Y & TAL Z & Side & Anatomic region & BA \\
\hline \multicolumn{8}{|c|}{ Pro-saccades } \\
\hline \multicolumn{8}{|c|}{ B pro-saccades versus pursuit } \\
\hline Size & $t \max$ & TAL X & TAL Y & TAL Z & Side & Anatomic region & BA \\
\hline \multicolumn{8}{|c|}{ Pro-saccades > pursuit } \\
\hline \multirow[t]{4}{*}{18,501} & 5.4 & $16 \pm 17$ & $-54 \pm 11$ & $44 \pm 5.8$ & Right & Inferior parietal lobule & 40 \\
\hline & & & & & Left/right & Superior parietal lobule including PEF & 7 \\
\hline & & & & & Left/right & Precuneus & 7 \\
\hline & 5.4 & 30 & -50 & 44 & Right & Superior parietal lobule & 7 \\
\hline \multirow[t]{2}{*}{7,359} & 5.6 & $-31 \pm 6.3$ & $-49 \pm 10$ & $39 \pm 7$ & Left & Inferior parietal lobule & 40 \\
\hline & & & & & & Superior parietal lobule including PEF & 7 \\
\hline \multirow[t]{6}{*}{7,348} & 5.9 & $-0.74 \pm 22$ & $-6.6 \pm 6.7$ & $51 \pm 4.8$ & Left/right & Medial frontal gyrus including SEF & 6 \\
\hline & & & & & Left/right & Anterior cingulate & 2432 \\
\hline & & & & & Left & Superior, middle frontal gyrus including FEF & 69 \\
\hline & 5.9 & 26 & -10 & 53 & Right & Middle frontal gyrus & 6 \\
\hline & 4.6 & -2 & -3 & 54 & Left/right & Medial frontal gyrus & 6 \\
\hline & 4.2 & -24 & -13 & 53 & Left & Middle frontal gyrus & 6 \\
\hline \multirow[t]{2}{*}{2,659} & 5.1 & $46 \pm 5.1$ & $-42 \pm 3.5$ & $12 \pm 4.2$ & Right & Superior temporal gyrus & 22 \\
\hline & & & & & & Middle temporal gyrus & 22 \\
\hline \multirow[t]{2}{*}{1,216} & 4.0 & $-49 \pm 2.7$ & $-44 \pm 5$ & $9.4 \pm 3$ & Left & Superior temporal gyrus & 22 \\
\hline & & & & & & Middle temporal gyrus & 21 \\
\hline 623 & 3.4 & $-29 \pm 3.6$ & $-78 \pm 5.4$ & $-5.3 \pm 2.2$ & Left & Lingual gyrus & 1819 \\
\hline 614 & 3.6 & $38 \pm 4.6$ & $-2.7 \pm 2.3$ & $34 \pm 3.6$ & Right & Precentral gyrus & 6 \\
\hline 535 & 4.2 & $34 \pm 2.4$ & $-45 \pm 3.5$ & $-18 \pm 1.6$ & Right & Fusiforme gyrus & 20 \\
\hline 479 & 4.6 & $-23 \pm 1.9$ & $-79 \pm 2.9$ & $-23 \pm 2.3$ & Left & Cerebellum & NA \\
\hline 290 & 3.8 & $-37 \pm 2.1$ & $39 \pm 1.7$ & $24 \pm 1.9$ & Left & Middle frontal gyrus & 10 \\
\hline 244 & 3.2 & $35 \pm 2.2$ & $-67 \pm 2.1$ & $15 \pm 1.7$ & Right & Middle temporal gyrus & 39 \\
\hline 234 & 3.3 & $16 \pm 2.4$ & $33 \pm 2$ & $30 \pm 1.7$ & Right & Medial frontal gyrus & 9 \\
\hline 219 & 3.3 & $-40 \pm 2.5$ & $8.7 \pm 1.5$ & $31 \pm 1.8$ & Left & Middle frontal gyrus & 9 \\
\hline 208 & 3.6 & $42 \pm 1.9$ & $-31 \pm 1.7$ & $0.62 \pm 1.5$ & Right & Superior temporal gyrus & 41 \\
\hline \multicolumn{8}{|c|}{ Pursuit $>$ pro-saccades } \\
\hline \multirow[t]{8}{*}{24,577} & -7.0 & $-0.87 \pm 8.8$ & $-68 \pm 10$ & $9.7 \pm 9.4$ & Left/right & Cuneus & 17181931 \\
\hline & & & & & Left/right & Precuneus & 31 \\
\hline & & & & & Left/right & Posterior Cingulate & 2330 \\
\hline & & & & & Left/right & Lingual gyrus & 1819 \\
\hline & & & & & Left/right & Cerebellum & NA \\
\hline & -6.9 & 0 & -78 & 28 & Left/right & Cuneus & 19 \\
\hline & -6.8 & -8 & -62 & 8 & Left & Cuneus & 31 \\
\hline & -6.6 & 10 & -69 & 8 & Right & Cuneus & 31 \\
\hline
\end{tabular}

Size of activation cluster in $\mathrm{mm}^{3}$, max $t$ value, center of gravity in Talairach space \pm standard deviation, anatomic region and Brodmann Area (BA). Local maxima are provided for large activation clusters if applicable in italics

work including bilateral cuneus, precuneus, posterior cingulate and lingual gyrus. Additional activations were present in bilateral posterior cerebellum. These patterns of activation are in close agreement with published results (Petit et al. 1997; Kimmig et al. 1999).
Corrective saccades during fixation, pro-saccades and pursuit

Activations associated with corrective saccades are illustrated in Fig. 3 and Table 2. 
Table 2 Activation clusters for CS, CF and CP (otherwise as in Table 1)

\begin{tabular}{|c|c|c|c|c|c|c|c|}
\hline \multicolumn{8}{|c|}{ Corrective saccades } \\
\hline \multirow{2}{*}{\multicolumn{4}{|c|}{$\begin{array}{lccc}\text { Size } \quad t \max \quad \text { TAL } X & \text { TAL Y } \\
\text { A corrective saccades during pro-saccades }-C S\end{array}$}} & \multirow[t]{2}{*}{ TAL Z } & \multirow[t]{2}{*}{ Side } & \multirow[t]{2}{*}{ Anatomic region } & \multirow[t]{2}{*}{ BA } \\
\hline & & & & & & & \\
\hline \multirow{9}{*}{27,041} & 6.2 & $5.5 \pm 11$ & $-71 \pm 9.3$ & $-0.38 \pm 12$ & Left/right & Cuneus & 1718 \\
\hline & & & & & & Lingual gyrus & 171819 \\
\hline & & & & & & Parahippocampal gyrus & 30 \\
\hline & & & & & & Cerebellum & NA \\
\hline & 6.2 & 3 & -76 & 14 & Right & Cuneus & 18 \\
\hline & 5.3 & -9 & -67 & -15 & Left & Cerebellum & $N A$ \\
\hline & 4.9 & 3 & -77 & 27 & Right & Cuneus & 18 \\
\hline & 4.9 & 28 & -74 & -15 & Right & Cerebellum & $N A$ \\
\hline & 4.8 & 9 & -70 & -11 & Right & Cerebellum & $N A$ \\
\hline \multirow[t]{8}{*}{15,782} & 5.0 & $36 \pm 17$ & $-47 \pm 12$ & $32 \pm 19$ & Right & Precuneus & 7 \\
\hline & & & & & & Superior parietal lobule including PEF & 7 \\
\hline & & & & & & Inferior parietal lobule & 40 \\
\hline & & & & & & Superior temporal gyrus & 22 \\
\hline & & & & & & Middle temporal gyrus & 2122 \\
\hline & 5.0 & 36 & -42 & 40 & Right & Inferior parietal lobule & 40 \\
\hline & 4.8 & 51 & -36 & 22 & Right & Superior temporal gyrus & 22 \\
\hline & 4.6 & 50 & -42 & 3 & Right & Middle temporal gyrus & 21 \\
\hline \multirow[t]{9}{*}{10,933} & 4.7 & $41 \pm 7.8$ & $16 \pm 14$ & $19 \pm 17$ & Right & Superior, middle frontal gyrus including FEF & 68910 \\
\hline & & & & & & Precentral gyrus & 46 \\
\hline & & & & & & Inferior frontal gyrus & 4445 \\
\hline & & & & & & Insula & 13 \\
\hline & & & & & & Superior temporal gyrus & 22 \\
\hline & 4.7 & 45 & 5 & 36 & Right & Middle frontal gyrus & 9 \\
\hline & 4.2 & 33 & 19 & 6 & Right & Anterior insula & 13 \\
\hline & 4.1 & 33 & 27 & 37 & Right & Middle frontal gyrus & 9 \\
\hline & 3.9 & 28 & 44 & -4 & Right & Middle frontal gyrus & 10 \\
\hline \multirow[t]{7}{*}{8,774} & 4.8 & $-47 \pm 7.9$ & $-47 \pm 8$ & $26 \pm 14$ & Left & Superior parietal lobule including PEF & 7 \\
\hline & & & & & & Inferior parietal lobule & 40 \\
\hline & & & & & & Superior temporal gyrus & 22 \\
\hline & & & & & & Middle temporal gyrus & 21 \\
\hline & 4.8 & -51 & -40 & 35 & Left & Inferior parietal lobule & 40 \\
\hline & 4.3 & -37 & -53 & 39 & Left & Inferior parietal lobule & 40 \\
\hline & 4.1 & -52 & -40 & 21 & Left & Superior temporal gyrus & 22 \\
\hline \multirow[t]{2}{*}{6,337} & 4.9 & $0.53 \pm 3.8$ & $0.85 \pm 9.9$ & $50 \pm 8.4$ & Left/right & Medial frontal gyrus including SEF & 6 \\
\hline & & & & & & Cingulate gyrus & 2432 \\
\hline \multirow[t]{3}{*}{2,011} & 4.9 & $-46 \pm 4$ & $7.5 \pm 3.8$ & $2.4 \pm 3.4$ & Left & Inferior frontal gyrus & 4445 \\
\hline & & & & & & Insula & 13 \\
\hline & & & & & & Superior temporal gyrus & 22 \\
\hline 1,676 & 4.2 & $-29 \pm 3.9$ & $-62 \pm 5.9$ & $-20 \pm 2.9$ & Left & Cerebellum & 0 \\
\hline 752 & 3.6 & $-36 \pm 3$ & $48 \pm 3$ & $2.3 \pm 2.3$ & Left & Middle frontal gyrus & 10 \\
\hline 586 & 3.6 & $30 \pm 2.1$ & $44 \pm 1.9$ & $13 \pm 4.5$ & Right & Middle frontal gyrus & 10 \\
\hline 495 & 4.0 & $37 \pm 2.2$ & $-5 \pm 2.8$ & $-7.5 \pm 2.1$ & Right & Insula & 13 \\
\hline \multirow[t]{2}{*}{403} & 3.3 & $-39 \pm 3.4$ & $1.5 \pm 2.3$ & $34 \pm 2.8$ & Left & Middle frontal gyrus & 9 \\
\hline & & & & & & Precentral gyrus & 46 \\
\hline
\end{tabular}


Table 2 continued

\begin{tabular}{|c|c|c|c|c|c|c|c|}
\hline \multicolumn{8}{|c|}{ Corrective saccades } \\
\hline Size & $t \max$ & TAL X & TAL Y & TAL Z & Side & Anatomic region & BA \\
\hline \multicolumn{8}{|c|}{ A corrective saccades during pro-saccades-CS } \\
\hline \multirow[t]{2}{*}{357} & 3.4 & $-34 \pm 2.6$ & $-11 \pm 2$ & $49 \pm 2.3$ & Left & Precentral gyrus & 4 \\
\hline & & & & & Left & Middle frontal gyrus & 6 \\
\hline \multirow[t]{2}{*}{338} & 3.9 & $-59 \pm 1.8$ & $-20 \pm 2.1$ & $14 \pm 2.2$ & Left & Transverse temporal gyrus & 41 \\
\hline & & & & & & Superior Temporal gyrus & 42 \\
\hline 300 & 3.4 & $-41 \pm 2.2$ & $-30 \pm 1.8$ & $12 \pm 1.8$ & Left & Transverse temporal gyrus & 41 \\
\hline \multirow[t]{2}{*}{278} & 3.7 & $-30 \pm 1.7$ & $-27 \pm 2.1$ & $13 \pm 2.4$ & Left & Insula & 13 \\
\hline & & & & & & Transverse temporal gyrus & 41 \\
\hline 269 & 3.3 & $1.8 \pm 1.5$ & $29 \pm 2.6$ & $29 \pm 2.1$ & Right & Cingulate gyrus & 32 \\
\hline \multicolumn{8}{|c|}{$\mathrm{B}$ corrective saccades during fixation- $\mathrm{CF}$} \\
\hline 5,542 & 5.9 & $0.28 \pm 7.3$ & $-75 \pm 6.5$ & $4.7 \pm 3.9$ & Left/right & Lingual gyrus & 1718 \\
\hline 1,310 & 4.4 & $13 \pm 3.7$ & $-56 \pm 3$ & $3.2 \pm 4.5$ & Right & Lingual gyrus & 19 \\
\hline 1,289 & 5.3 & $-48 \pm 4.4$ & $-54 \pm 4.9$ & $11 \pm 2.7$ & Left & Middle temporal gyrus & 39 \\
\hline 702 & 4.7 & $-48 \pm 3.7$ & $10 \pm 3.3$ & $-1 \pm 2.1$ & Left & Insula & 13 \\
\hline 476 & 4.1 & $-51 \pm 1.5$ & $-38 \pm 2.5$ & $27 \pm 4.8$ & Left & Inferior parietal lobule & 40 \\
\hline 250 & 3.6 & $-44 \pm 1.4$ & $5.5 \pm 3.4$ & $37 \pm 3.9$ & Left & Middle frontal gyrus & 89 \\
\hline 213 & 4.0 & $3 \pm 1.4$ & $-9.2 \pm 1.5$ & $53 \pm 2.3$ & Right & Medial frontal gyrus & 6 \\
\hline 203 & 4.0 & $-21 \pm 1.9$ & $-11 \pm 1.5$ & $59 \pm 1.7$ & Left & Middle frontal gyrus including FEF & 6 \\
\hline \multicolumn{8}{|c|}{$\mathrm{C}$ corrective saccades during pursuit $-\mathrm{CP}$} \\
\hline \multicolumn{8}{|c|}{ No suprathreshold activations } \\
\hline
\end{tabular}

Size of activation cluster in $\mathrm{mm}^{3}$, max $t$ value, center of gravity in Talairach space \pm standard deviation, anatomic region and Brodmann Area (BA). Local maxima are provided for large activation clusters if applicable in italics

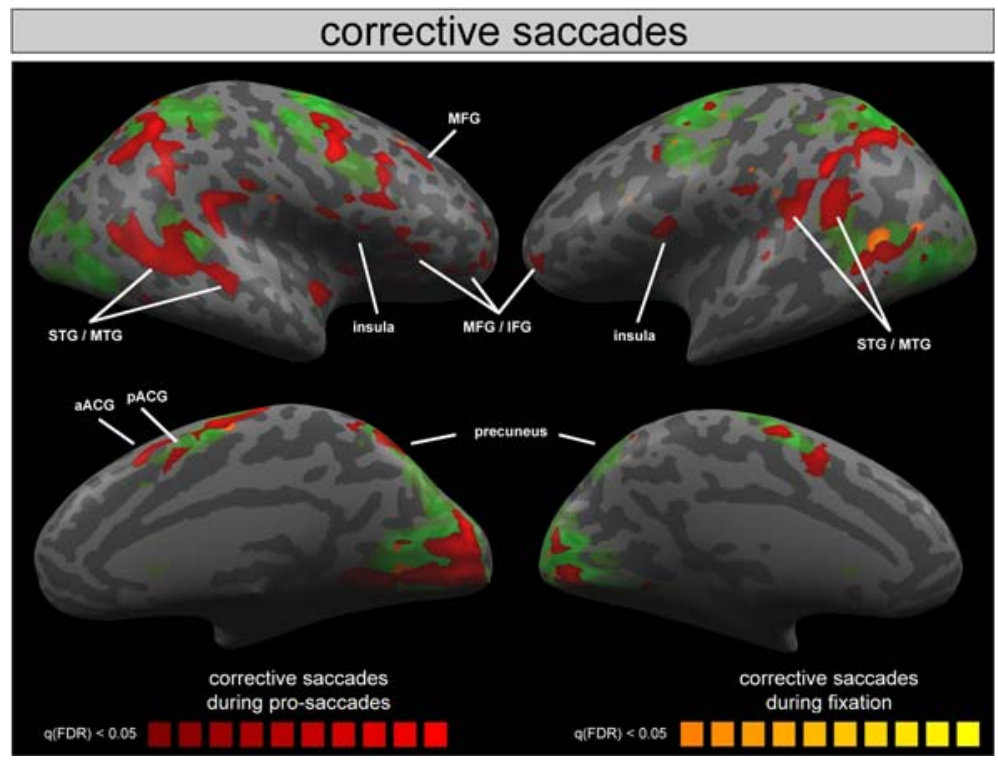

Fig. 3 illustrates activations associated with corrective saccades during pro-saccades (CS, red) and fixation ( $\mathrm{CF}$, orange-yellow). The activations of pro-saccades are superimposed for means comparison in green. CS evoked activations in the prosaccades network and additional activations in anterior inferior cingulate and bilateral middle and inferior frontal gyri and bilateral insula. CF yielded activations domi- nantly within the pursuit network and additionally in left insula. No supra-threshold activations were present for corrective saccades during pursuit (CP). Essential anatomic regions are labelled: $M F G$ middle frontal gyrus, $I F G$ inferior frontal gyrus, $S T G$ superior temporal gyrus, $M T G$ middle temporal gyrus, $a A C G$ anterior aspect of anterior cingulate gyrus, $p A C G$ posterior aspect of anterior cingulate gyrus 
Corrective saccades occurring during pro-saccades (CS) were associated with a distributed network of activations, which largely overlaps with the pro-saccade network. Activations in additional areas were present in anterior-inferior cingulate and bilateral middle and inferior frontal gyri and bilateral anterior insula (Table 2).

Corrective saccades during fixation (CF) yielded activations within the pro-saccades network (notably bilateral lingual gyrus, left middle frontal gyrus including FEF and left middle temporal gyrus). Additional activations were found in left insula.

Corrections saccades during pursuit (CP) did not evoke any supra-threshold activations. The reasons for this lack of $\mathrm{CP}$ activation are discussed below.

\section{Discussion}

The combination of functional magnetic resonance imaging (fMRI) and MR compatible eye tracking was implemented to assess the neuronal circuitry mediating the execution of corrective saccades. More specifically, neuronal activations associated with corrective saccades were related to the modification of the neuronal activations associated with pro-saccades and pursuit eye movement. In accordance to our hypothesis, we demonstrated specific neuronal activations associated with corrective saccades in addition to common neuronal activations evoked by pro-saccades and, to a lesser extent, by pursuit eye movements.

Pro-saccades and pursuit eye movement

In a first step, we assessed neuronal activations associated with the execution of pro-saccades (S) and pursuit eye movement $(\mathrm{P})$ in a block-design, which served as the basis for the later comparison with corrective saccades. In line with previous investigations, $\mathrm{S}$ and $\mathrm{P}$ yielded activations in largely overlapping networks including SEF, FEF and PEF (Berman et al. 1999; Petit and Haxby 1999) and the occipito-temporal junction (Petit and Haxby 1999), which is considered as human homologue of monkey MT/MST (Zeki et al. 1991; Watson et al. 1993).

$\mathrm{S}$ compared to $\mathrm{P}$ evoked stronger activations in several areas defined in the analysis of S, notably SEF, FEF and PEF, in line with a previous study (Petit and Haxby 1999). In another study, these areas had a trend to greater activations during $\mathrm{S}$ compared to $\mathrm{P}$, which was significant only in FEF (Berman et al. 1999). In contrast to these investigations, we compared the magnitude of activations in the $S$ and $P$ conditions using statistical parametric mapping. This revealed additional activations for $\mathrm{S}$ versus $\mathrm{P}$ in bilateral middle frontal gyri, left inferior frontal gyrus and left ante- rior insula. It was shown recently that these areas are active in visually and memory guided saccade tasks (Brown et al. 2004; Ozyurt et al. 2006).

The inverse comparison $\mathrm{P}$ versus $\mathrm{S}$ yielded stronger activations in cuneus, precuneus (Berman et al. 1999; Petit and Haxby 1999), lingual gyrus and posterior cingulate (Berman et al. 1999). Correspondingly, the direct comparison of $P$ versus $S$ yielded additional activations in bilateral cerebellum. This region has been shown to be involved in the control of closed-loop, oculo-motor control of pursuit (O’Driscoll et al. 2000).

\section{Corrective saccades}

In contrast to previous investigations (Berman et al. 1999; Petit and Haxby 1999), we assessed eye movements during the fMRI experiments using an MR-compatible eye tracker (Kimmig et al. 1999). This allows for an event-related analysis of neuronal activations associated with corrective saccades.

The frequency of spontaneously occurring corrective saccades differed significantly depending on the performed oculomotor task. The frequency of spontaneous corrective saccades increased from fixation across pro-saccades to pursuit eye movement.

The most pronounced activations associated with corrective saccades were present during pro-saccades (CS). The resulting activation network largely overlapped with the activation network defined for pro-saccades, yet revealed additional activations in anterior inferior cingulate and bilateral middle and inferior frontal gyri and bilateral-anterior insula. This implies a specific role of these above-mentioned areas in the execution of corrective saccades. These areas have not been reported in previous investigations, which assessed various aspects of the neural control of saccades including intentional saccades, predictive saccades, memory guided saccades, anti-saccades or pro-saccades (for a review, Pierrot-Deseilligny et al. 2004) and were accordingly not active in the pro-saccades condition of the presented study. It should be mentioned however that some previous studies have focused on the established neural circuitry that controls the oculomotor system (e.g., SEF, FEF and PEF) and did not investigate the whole brain. In particular, middle and inferior frontal gyri were not covered in the investigated volume in some studies (Berman et al. 1999; Kimmig et al. 2001).

Activations in the posterior aspect of the anterior cingulate gyrus (pACG), which has also been called the 'cingulate eye field' (Pierrot-Deseilligny et al. 2004), and activations in posterior cingulate gyrus (PCG) are known to be associated with saccade control (Paus et al. 1993; Nobre et al. 1997; Gaymard and Pierrot-Deseilligny 1999; PierrotDeseilligny et al. 2004). In agreement with these investigations, pACG and PCG were active during S and CS in the 
present study. The anterior-inferior aspect of the anterior cingulate cortex (aACG), in contrast, was active only during CS. The activations in aACG and bilateral inferior frontal gyri/bilateral anterior insula are very similar to those reported in an fMRI study, which investigated recenteringsaccades (Raemaekers et al. 2005). Recentering-saccades direct the gaze back to the center of the display, following e.g., pro-saccades and anti-saccades. We suggest that these recentering-saccades share fundamental similarities with corrective saccades because both forms of saccades redirect the gaze. During the central fixation periods between two consecutive pro-saccades, i.e., for approximately 50\% of the time in the $\mathrm{S}$ condition, corrective saccades actually redirect gaze to the central visual target and hence are very similar to the above-mentioned recentering-saccades.

Corrective saccades during fixation (CF) evoked similar yet less pronounced activations. This can be attributed to the lower frequency of corrective saccades during fixations as compared to during pro-saccades. Activations were predominantly evident within the pro-saccade network. Additional activation was present in left insula, in agreement with CS.

Corrective saccades during pursuit (CP) yielded no significant activations. The fact that $\mathrm{CP}$ yielded no suprathreshold activation in contrast to $\mathrm{CS}$ and $\mathrm{CF}$ appears contra-intuitive at first glance because corrective saccades occurred almost three times more frequently during pursuit (P) than during pro-saccades (S). If the occurrence of corrective saccades increases, the linear dependence between the block-design regressor, e.g., $\mathrm{P}$ and the event-related regressor $\mathrm{CP}$ increases and it becomes progressively less possible to differentiate neuronal activations associated with $\mathrm{P}$ and $\mathrm{CP}$. Note that the hemodynamic response function (HRF) (Friston et al. 1998) introduces an additional temporal filter, that causes similar regressors for $\mathrm{P}$ and $\mathrm{CP}$ given the high number of events. Under the assumption of this effect in particular in CP, we suggest that part of the activations in the established pro-saccade network during $\mathrm{P}$ may be related to corrective saccades rather than to pursuit per se. This consideration is supported by the observations that the amplitude of the BOLD response, in particular in FEF, increases almost linearly with increasing frequency of the pro-saccades while the amplitude of pro-saccades is irrelevant (Kimmig et al. 2001). If this observation can be transferred to corrective saccades, this implies that only the frequency of corrective saccades is of importance while their (small) amplitude is irrelevant. This suggests that the high number of spontaneously occurring corrective saccades during $\mathrm{P}$ should evoke pronounced activations, for e.g., in FEF. In line with this argumentation, it was suggested that FEF activation might be related to corrective saccades, which has been shown to occur during erroneous anti-saccades in a randomized pro- and anti-saccade task (Cornelissen et al. 2002).
The presented results imply that corrective saccades represent a potential systematic confound for the interpretation of the neuronal correlates of oculomotor control, in particular if eye movements are not measured and corrective saccades are not included in the GLM analysis. This concern is in particular supported by the fact that the spontaneous occurrence of corrective saccades depends on the oculomotor task.

\section{Limitations}

The major limitation of the present investigation resides in the nature of corrective saccades, which occur spontaneously and by definition only together with on-going eye movements. This complicates the separation of neuronal activations related to corrective saccades and the neuronal activations associated with, e.g., pursuit per se. The involuntary occurrence renders impossible standard fMRI ONOFF designs and/or systematic parametric variation in task difficulty, which would represent one possibility to overcome this limitation (Amaro and Barker 2006). We therefore modeled the basic eye movement condition as nonexplanatory regressors. Ideally, variance in BOLD response associated with the primary eye movement condition should be represented in the corresponding regressor. The remaining variance in BOLD response, which is associated with the behavioral occurrence of corrective saccades, presumably reflects neuronal activations due to corrective saccades. The presented results regarding the neuronal correlates of corrective saccades should therefore be considered with caution given the intrinsic difficulty of separating the effects of corrective saccades from those of the on-going oculomotor task. Notwithstanding, we argue that corrective saccades are a fundamental component of eye movement control, which implies that some methodological constraints must be taken into account. Under the assumption that the occurrence of corrective saccades depends on the difficulty of the on-going eye-movement task, one possible amendment for future studies would reside in the titration of the eye movement task difficulty to evoke the frequency of corrective saccades, which is optimal for separation of eye movement-related and corrective saccades-related neuronal activations. For example, the occurrence of corrective saccades during smooth pursuit is lower for slower targets and for sinusoidal rather than constant velocity movement (Berman et al. 1999).

In addition to the frequency, the amplitude of corrective saccades might be of interest. In principle, it is possible to estimate the amplitude of eye movements with the used MR eye-tracker. The MR eye-tracker sensor is attached to the head coil. Consequently, head motion causes a shift of the center of gaze relative to this camera. We compared the measured eye position to the known stimulus position. We 
do not report the corrective saccades amplitude results because in some volunteers, even (inevitable) small head motion evoked substantial and non-linear mis-estimation of the eye-positions in relation to the relatively small amplitude of corrective saccades. It has, however, been demonstrated that the amplitude of pro-saccades is irrelevant for the BOLD activations while the frequency of pro-saccades almost linearly correlates with the BOLD response (Kimmig et al. 2001). Although this observation was made for pro-saccades and not for corrective saccades, this finding at least suggests that the amplitude of corrective saccades is less relevant than the frequency. The frequency could be determined with high accuracy with the MR eye-tracker.

Given these methodological constraints of the analysis of BOLD activations associated with corrective saccades, as discussed above, we cannot rule out that hypometria of the primary saccades and not necessarily the performance of corrective saccades per se might at least contribute to the observed activations of CS. One possibility to validate the presented results would be a parametric analysis of other ocular motor parameters such as saccade gain or saccade dysmetria, which might support a specific association of the reported activations with the performance of corrective saccades. At least in our experimental setup, the amplitude of the corrective saccades is however small in relation to the accuracy of the estimated eye position, as discussed above. This impedes a reliable parametric analysis of our data, but might be an option in a future setup. As discussed above, the temporal estimation of the onset of corrective saccades, in contrast, was possible at high accuracy.

We chose another approach to validate the presented results and reanalyzed the data with randomly varied onsets of corrective saccades, creating pseudo-corrective saccades event-related regressors. The block-design regressors for pro-saccades and pursuit were unchanged. As expected, the resulting activation patterns for the pseudo-corrective saccades differed between different variants of this random analysis, while the block-design regressors remained largely constant. This supports the assumption that the reported results are specifically related to the onset of corrective saccades, and illustrates the power of GLM analyses to separate the underlying task (here e.g., pro-saccades) from additional events (here e.g., corrective saccades during pro-saccades).

\section{Perspectives}

The present investigation provides functional MRI results concerning the neural control of corrective saccades. We demonstrate the feasibility of assessing eye movements and corrective saccades during fMRI and illustrate that corrective saccades represent a putative systematic confound in functional imaging of eye movements. At this stage, we could not directly compare BOLD activations of corrective saccades arising in the $\mathrm{CS}$ and $\mathrm{CP}$ conditions, owing to differences in the spontaneous frequency of the events. We found substantial and significant difference in the frequency of corrective saccades between these conditions, which would represent a systematic confound in the direct comparison. Consequently, the interpretation of these difference analyses is problematic. It is difficult to control the frequency of corrective saccades, as these are involuntary events. As discussed above, it might be possible to influence the frequency of corrective saccades by modifying the difficulty of the eye-movement task. Corrective saccades can be directly compared in future studies only if it is possible to achieve similar frequencies of corrective saccades in the different conditions. Additionally, we did not differentiate corrective saccades into sub-types. For example, corrective saccades during pursuit are also called catch-up saccades and it might physiologically be relevant to discriminate catch-up saccades in $\mathrm{ON}$ direction (i.e., the direction of pursuit) and OFF direction (i.e., in the opposite direction of pursuit). Likewise, during pro-saccades, it might be relevant to discriminate corrective saccades towards the center versus towards the periphery. Furthermore single corrective saccades might be differentiated from multiple corrective saccades. Subdivision of corrective saccades on the one hand reduces the number of events per condition and on the other hand requires additional predictors/regressors in the GLM analysis. Both modifications likely reduce the level of significance of the fMRI activations. Specific assessment of these sub-types of corrective saccades must be performed in future, specifically tailored investigations.

\section{Conclusion}

We demonstrated a neuronal network that is most likely specifically associated with corrective saccades and partially overlaps with the established pro-saccade and pursuit eye movement networks. The presented results suggest that corrective saccades represent a potential systematic confound in eye-movement studies in particular because the frequency of spontaneously occurring corrective saccades depends on the underlying oculomotor task.

\section{References}

Amaro E Jr, Barker GJ (2006) Study design in fMRI: basic principles. Brain Cogn 60:220-232

Belliveau JW, Kwong KK, Kennedy DN, Baker JR, Stern CE, Benson R, Chesler DA, Weisskoff RM, Cohen MS, Tootell RB et al (1992) Magnetic resonance imaging mapping of brain function. Human visual cortex. Invest Radiol 27(Suppl 2):S59-S65 
Bennett SJ, Barnes GR (2006) Combined smooth and saccadic ocular pursuit during the transient occlusion of a moving visual object. Exp Brain Res 168:313-321

Berman RA, Colby CL, Genovese CR, Voyvodic JT, Luna B, Thulborn KR, Sweeney JA (1999) Cortical networks subserving pursuit and saccadic eye movements in humans: an FMRI study. Hum Brain Mapp 8:209-225

Boynton GM, Engel SA, Glover GH, Heeger DJ (1996) Linear systems analysis of functional magnetic resonance imaging in human V1. J Neurosci 16:4207-4221

Brown MR, DeSouza JF, Goltz HC, Ford K, Menon RS, Goodale MA, Everling S (2004) Comparison of memory- and visually guided saccades using event-related fMRI. J Neurophysiol 91:873-889

Cornelissen FW, Kimmig H, Schira M, Rutschmann RM, Maguire RP, Broerse A, Den Boer JA, Greenlee MW (2002) Event-related fMRI responses in the human frontal eye fields in a randomized pro- and antisaccade task. Exp Brain Res 145:270-274

Dodge R (1903) Five types of eye movement in the horizontal meridian plane of the field of regard. Am J Physiol 8:307-329

Friston KJ, Holmes AP, Worsley KJ, Poline JB, Frith CD, Frackowiak RSJ (1995) Statistical parametric maps in functional imaging: a general linear approach. Hum Brain Mapp:189-210

Friston KJ, Fletcher P, Josephs O, Holmes A, Rugg MD, Turner R (1998) Event-related fMRI: characterizing differential responses. Neuroimage 7:30-40

Gaymard B, Pierrot-Deseilligny C (1999) Neurology of saccades and smooth pursuit. Curr Opin Neurol 12:13-19

Genovese CR, Lazar NA, Nichols T (2002) Thresholding of statistical maps in functional neuroimaging using the false discovery rate. Neuroimage 15:870-878

Huey EB (1900) On the psychology and physiology of reading. Am J Psychol 11:283-302

Kimmig H, Greenlee MW, Huethe F, Mergner T (1999) MR-eyetracker: a new method for eye movement recording in functional magnetic resonance imaging. Exp Brain Res 126:443-449

Kimmig H, Greenlee MW, Gondan M, Schira M, Kassubek J, Mergner $\mathrm{T}$ (2001) Relationship between saccadic eye movements and cortical activity as measured by fMRI: quantitative and qualitative aspects. Exp Brain Res 141:184-194
Nobre AC, Sebestyen GN, Gitelman DR, Mesulam MM, Frackowiak RS, Frith CD (1997) Functional localization of the system for visuospatial attention using positron emission tomography. Brain 120(Pt 3):515-533

O'Driscoll GA, Wolff AL, Benkelfat C, Florencio PS, Lal S, Evans AC (2000) Functional neuroanatomy of smooth pursuit and predictive saccades. Neuroreport 11:1335-1340

Ogawa S, Lee TM, Nayak AS, Glynn P (1990) Oxygenation-sensitive contrast in magnetic resonance image of rodent brain at high magnetic fields. Magn Reson Med 14:68-78

Ozyurt J, Rutschmann RM, Greenlee MW (2006) Cortical activation during memory guided saccades. Neuroreport 17:1005-1009

Paus T, Petrides M, Evans AC, Meyer E (1993) Role of the human anterior cingulate cortex in the control of oculomotor, manual, and speech responses: a positron emission tomography study. J Neurophysiol 70:453-469

Petit L, Haxby JV (1999) Functional anatomy of pursuit eye movements in humans as revealed by fMRI. J Neurophysiol 82:463471

Petit L, Clark VP, Ingeholm J, Haxby JV (1997) Dissociation of saccade-related and pursuit-related activation in human frontal eye fields as revealed by fMRI. J Neurophysiol 77:3386-3390

Pierrot-Deseilligny C, Milea D, Muri RM (2004) Eye movement control by the cerebral cortex. Curr Opin Neurol 17:17-25

Pola J, Wyatt HJ (1991) Smooth pursuit: response characteristics, stimuli and mechanisms. Macmillan, London

Raemaekers M, Vink M, van den Heuvel MP, Kahn RS, Ramsey NF (2005) Brain activation related to retrosaccades in saccade experiments. Neuroreport 16:1043-1047

Talairach J, Tournoux P (1988) Co-planar stereotaxic atlas of the human brain. Thieme, New York

Watson JD, Myers R, Frackowiak RS, Hajnal JV, Woods RP, Mazziotta JC, Shipp S, Zeki S (1993) Area V5 of the human brain: evidence from a combined study using positron emission tomography and magnetic resonance imaging. Cereb Cortex 3:79-94

Yarbus AL (1967) Eye movements during perception of complex objects. Plenum Press, New York

Zeki S, Watson JD, Lueck CJ, Friston KJ, Kennard C, Frackowiak RS (1991) A direct demonstration of functional specialization in human visual cortex. J Neurosci 11:641-649 